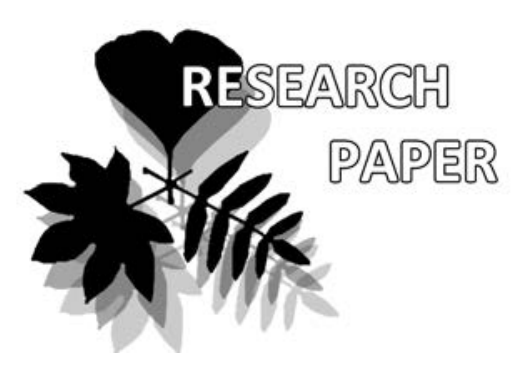

\title{
Endemic Quercus pontica C. Koch. communities from the Colchic Province and new syntaxonomical concept for the Caucasian subalpine krummholz vegetation
}

\author{
Nikolai B. Ermakov ${ }^{1,3,4}$, Yuriy V. Plugatar ${ }^{1} \&$ Vitaliy D. Leiba ${ }^{2}$
}

Nikolai B. Ermakov ${ }^{1,3,4 *}$

e-mail: brunnera@mail.ru

Yuriy V. Plugatar ${ }^{1}$

e-mail: plugatar.y@gmail.com

Vitaliy D. Leiba ${ }^{2}$

e-mail:abnilos@rambler.ru

${ }^{1}$ Nikitsky Botanical Garden - National Scientific Center RAS, Yalta, Nikita,

Crimea Republic, Russia

${ }^{2}$ Abkhazian Experimental Research Forest Station, Ochamchura, Abkhazia

${ }^{3}$ Maykop State Technological University, Maykop, Adygeya Republic, Russia

${ }^{4}$ Khakassia State University, Abakan, Khakassia Republic, Russia

\section{* corresponding author}

Manuscript received: 03.05.2020

Review completed: 28.05.2020

Accepted for publication: 05.06 .2020

Published online: 11.06 .2020

\begin{abstract}
A B S T R A C T
As a result of the classification of the Quercus pontica endemic communities from the Western Caucasus and subsequent comparative analysis with published data, we described new syntaxa and revised the existing concept of Caucasian krummholz higher units. The Rhododendro lutei-Quercetum ponticae ass. nova hoc loco, Corylo avellanae-Quercetum ponticae ass. nova hoc loco and Querco ponticae-Betuletum medwediewii Eminagaoglu et al. 2006 were united into the Quercion ponticae all. nova hoc loco. The Senecioni-Betuletum litwinowii Onipchenko 2002 and Astrantio-Betuletum litwinowiz Sokolova ex hoc loco were united in the Acero trantwetteri-Betulion litwinowii all. nova hoc loco. Both alliances were included into the order Acero trantvetteri-Betuletalia litwinowii ord. nova hoc loco, the class Betulo-Alnetea viridis Rejmanek ex Boeuf et al. 2014. The order Rhododendro caucasicae-Betuletalia litwinowii Mucina 2016 (and alliance Rhododendro-Betuletalia litwinowii Onipchenko 2002) should be assigned to the class Vaccinio-Piceetea Br.-Bl. in Br.-Bl. et al. 1939.

Ke y w o r d s : subalpine vegetation, Quercus pontica, classification, endemic plant communities, Caucasus
\end{abstract}

\section{P E 3 Ю M E}

Ермаков Н.Б., Пиугатарь Ю.В., Аейба В.А. Эндемичные сообщества Quercus pontica C. Косh. из КолхиАской провинции и новая концепция синтаксономии субальпийского криволесья Кавказа. В результате проведенной классификации эндемичных сообществ с Аоминированием дуба понтийского (Quercus pontica) Колхилской провинции (Западный Кавказ) и сравнительного анализа с опубликованными Аанными по субальпийскому криволесью Кавказа были описаны новые синтаксоны и проведена ревизия существующей концепции высших еАиниц. Ассоциации Rhododendro luteiQuercetum ponticae ass. nova hoc loco, Corylo avellanae-Quercetum ponticae ass. nova hoc loco, Querco ponticae-Betuletum medwediewii Eminagaoglu et al. 2006 были объединены в союз Quercion ponticae all. nova hoc loco. Ассоциации Senecioni-Betuletum litwinowii Onipchenko 2002 и Astrantio-Betuletum litwinowii Sokolova ex hoc loco были объединены в союз Acero trautvetteri-Betulion litwinowii all. nova hoc loco. Оба союза включены в порядок Acero trantvetteri-Betuletalia litwinowii ord. nova hoc lосо, в класс Betulo-Alnetea viridis Rejmanek ex Boeuf et al. 2014. Порядок Rhododendro caucasicae-Betuletalia litwinowii Mucina 2016 (и союз RhododendroBetuletalia litwinowii Onipchenko 2002) слелует рассматривать в классе VaccinioPiceetea Br.-Bl. in Br.-Bl. et al. 1939.

КАючевые слова: субальпийская растительность, Quercus pontica, классификация растительности, эндемичные растительные сообщества, Кавказ
The Caucasus is one of the largest and oldest mountain systems of the Western Palearctic whose geological age is determined from the Proterozoic. Like many ancient mountain systems in the temperate zone of Eurasia, the Caucasus is characterized by a high level of diversity and originality of flora and vegetation. One of the remarkable features of the Caucasus is the phenomenon of numerous endemic plants in the subalpine and alpine zones (Grossheim 1936, 1948, Kolakovsky 1961, Gagnidze 1974, Shetekauri et al. 2012). Some of them form peculiar plant communities at the upper boundary of the forest belt. The most distinctive examples are birches (Betula litwinowii Doluch., B. medwediewii Regel), as well as the unique subalpine Pontic oak Quercus pontica C. Koch. - creeping shrub (sometimes a low tree)
3-5 $\mathrm{m}$ tall, with big leaves (up to $35 \mathrm{~cm}$ long). This endemic oak species does not form a continuous range but locally occurs on the southern macro-slope of the western part of the Greater Caucasus, as well as in the south-western part of the Lesser Caucasus (Kolakovsky 1982). General (and very poor) information about the communities formed by Quercus pontica is available in some publications (Gulisashvili et al. 1975, Tumadzhanov 1980, Menitskiy 1982, Zazanashvili et al. 2000). Currently, there is only one publication on syntaxonomy of the birch-oak (Betula medwediewii, Quercus pontica) krummholz (Eminagaoglu 2006) from the upper part of the forest belt of the Lesser Caucasus. At the same time, similar Quercus pontica communities occurring in the subalpine zone of the Greater Caucasus have not yet been described. 
The paper aims on presentation of classification results and comparative syntaxonomic analysis of the subalpine Quercuspontica communities in the context of considering the problems of subalpine krummholz vegetation classification of the Caucasus as a whole.

\section{Study area}

The studied area is located in the subalpine zone of the Pskhu River basin (right tributary of the Bzyb river, Abkhazia) (Fig. 1). This territory is placed on the southern spurs of the Greater Caucasus ridge (the western part) at altitudes 1800-2300 $\mathrm{m}$ a.s.l. The mountain ranges are composed mainly of ancient crystalline rocks - gneisses, crystalline schists and granites. The relief is characterized by very steep mountain slopes with slow erosion processes, open outcrops and narrow gorges (Kamanin et al. 1974, Antonov et al. 1977). The climate at an altitude of about $2000 \mathrm{~m}$ is moderately cold and very humid. The average annual temperature is $3.9^{\circ} \mathrm{C}$. The average temperature of the warmest month (August) is $10-12^{\circ} \mathrm{C}$, the coldest month (January) is $-6^{\circ}--8^{\circ} \mathrm{C}$. Winter lasts $5-6$ months but deep snow cover lasts longer. The average annual precipitation reaches 2000-2500 mm (Gvozdetsky 1963) that brought by active Mediterranean cyclones through the western part of the Caucasus. Snow cover reaches $4 \mathrm{~m}$ depth in winter

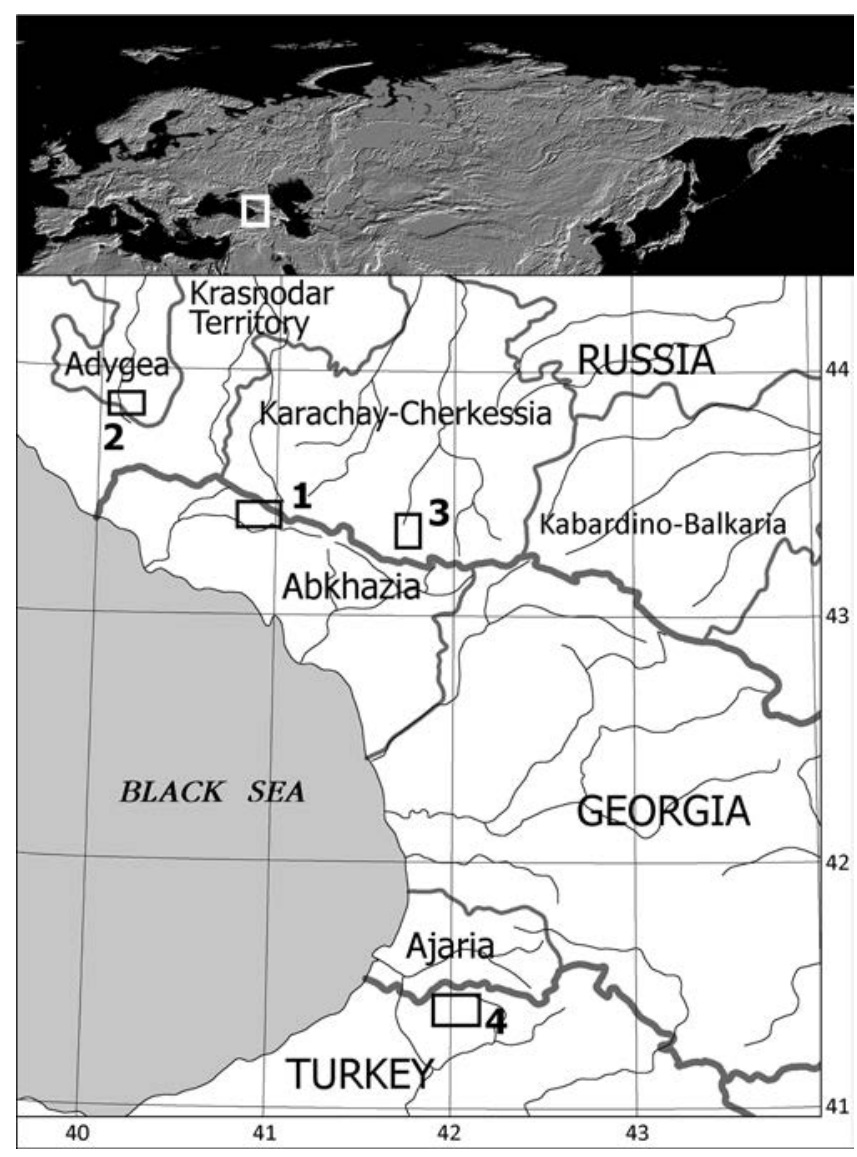

Figure 1 Geographical locations of relevés used for the syntaxonomic analysis: 1 - relevés made by Nikolai Ermakov in the Pskhu River Basin, Abkhazia (Western Caucasus), 2 - relevés from the upper part of the Belaya River Basin (North-Western Caucasus) (Sokolova 2013), 3 - releves from Teberda Nature Reserve (Northern Caucasus) (Onipchenko 2002), 4 - releves from the Lesser Caucasus (Eminagaoglu et al. 2006)
(Tephnadze et al. 2014). Therefore, avalanches play an important role in the formation of subalpine landscapes. The Quercus pontica communities are usually formed on very steep, well-warmed southern mountain slopes with outcrops of indigenous crystalline rocks and poorly developed soils.

\section{MATERIAL AND METHODS}

The database for the classification and comparative syntaxonomic analysis included 51 relevés of subalpine krummholz from various regions of the Caucasus. Fifteen relevés of communities dominated by Quercus pontica (Fig. 2A) were made by the authors during field studies in the subalpine belt and upper part of the forest belt on the southern macroslope of the Greater Caucasus (the Pskhu River Basin, Abkhazia) (Fig. 1, 2B). We also included in the analysis 20 relevés of Betula litwinowii communities from the subalpine zone of the Northern Caucasus (Teberda Nature Reserve) published by Onipchenko (2002, Table 13.1, relevé nrs 3, 4, 5, 11, 12, 22, 23, 26, 29, 46, 60, 62, $63,98,101,133,143,173,191,195), 10$ relevés of Betula medwediewii and Quercus pontica communities from the southern macroslope of the Lesser Caucasus published by Eminagaoglu et al. (2006, Table 1, relevés nr. 1-10) and 7 relevés of Betula litwinowii communities from the northern macroslope of the Western Caucasus (upper part of the Belaya River Basin) published by Sokolova (2013, Table 1, relevés nr. 1-7). All relevés were storied in the Turboveg database (Hennekens \& Schaminée 2001). The classification of the entire set of relevés was carried out in accordance with the Braun-Blanqet approach (Westhoff \& Maarel 1978). Syntaxa names were proposed according to the Code of Phytosociological Nomenclature (Weber et al. 2000). Quantitative classification of relevés was performed using cluster analysis (Ward method, Euclidian distance) implemented in the PC-Ord (McCune 2006). We followed Czerepanov (1995) and Ignatov et al. (2006) in vascular plants and bryophytes taxonomy.

\section{RESULTS}

As a result of the quantitative classification (Ward's method, Euclidian distance), all relevés were combined into 6 distinct clusters interpreted as associations in dendrogram (Fig. 3). Two of them, Corylo-Quercetum ponticae (I) and Rhododendro lutei-Quercetum ponticae (II) described from the southern macro-slope of the Greater Caucasus are new for science. The other four associations, Querco ponticaeBetuletum medwediewii (III), Senecioni nemorensis-Betuletum litwinowii (IV), Astrantio maximae-Betuletum litwinowii (V) and Rhododendro caucasici-Betuletum litwinowii (VI), represent the krummholz communities from the subalpine belt of the northern macro-slope of the Greater Caucasus (Onipchenko 2002, Sokolova 2013) and the southern macro-slope of the Lesser Caucasus (Eminagaoglu 2006). The relevés of these six associations were united at the next hierarchical level in three clusters interpreted as alliances (A - Quercion ponticae, B - Acero trautvetteri-Betulion litwinowii, C - Rhododendro caucasici-Betulion litwinowii) (Fig. 3). The results of cluster analysis have demonstrated high floristic integrity and originality of all six associations and 

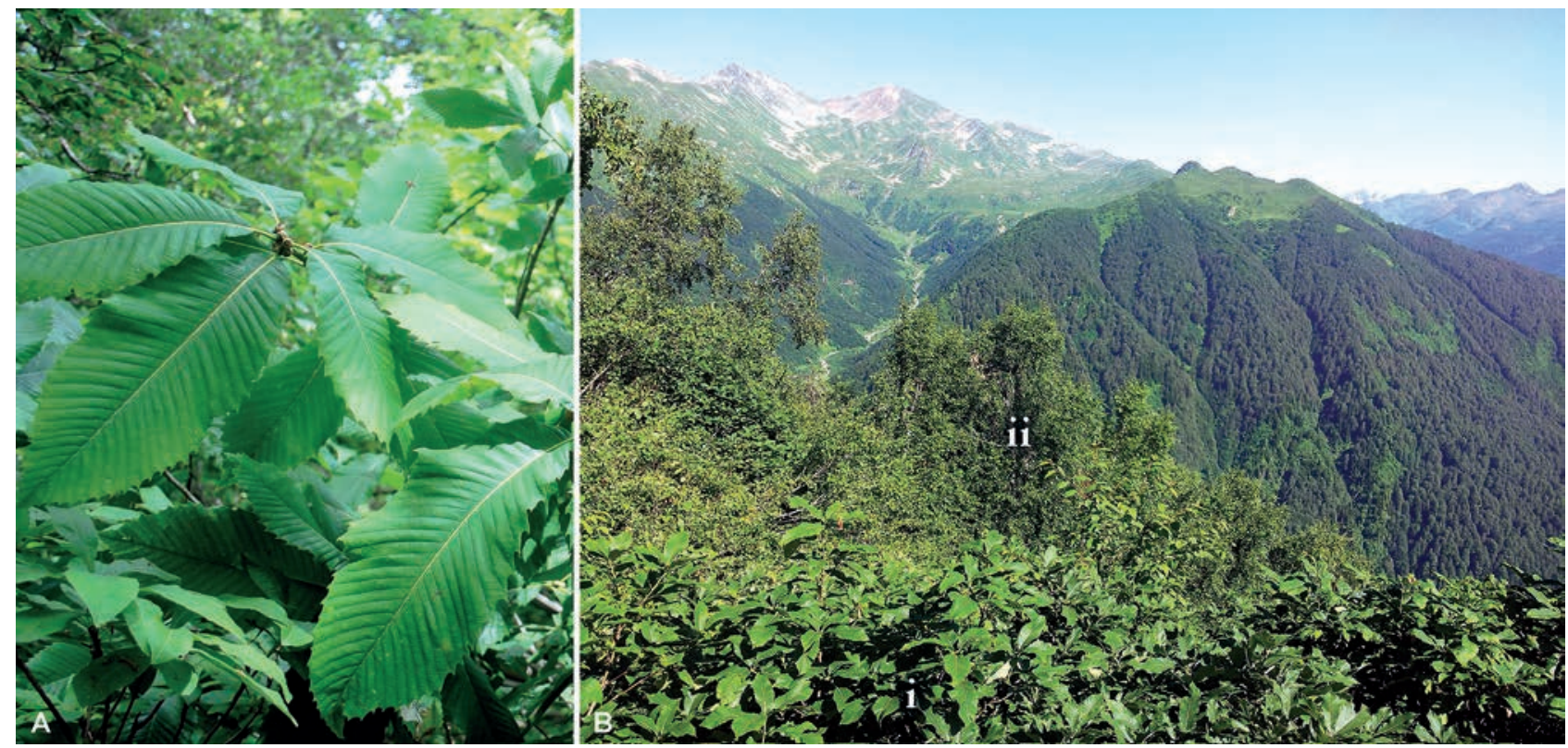

Figure 2 Quercus pontica C. Koch. communities in landscapes of Greater Caucacus. A - endemic Pontic oak Quercus pontica - creeping shrub (sometimes a low tree) 3-5 m tall, with big leaves (up to $35 \mathrm{~cm}$ long); it locally occurs in the Western Caucasus; B - subalpine Ouercus pontica krummholz (i) with Betula litwinowii Doluch. at the background (association Rhododendro lutei-Quercetum ponticae ass. nova hoc loco) (ii) occurring as small patches on very steep $\left(45-60^{\circ}\right)$ slopes at the upper boundary of the forest belt at altitudes $1800-2150 \mathrm{~m}$. Quercus pontica forms a very dense 2-3 m high shrub layer with a cover 90-100\%. Photo by Sergey Bebia in the upper reaches of the Pskhu River (right tributary of the Bzyb River, Abkhazia)

three alliances. However, their integrations at the highest hierarchical level of the dendrogram in two clusters $(\mathrm{Hl}$ and H2, Fig. 3) were determined to a greater extent by the high values of cover (predominance effect) of the three main dominants - Quercus pontica, Betula litwinowii, B. medwediewii but not by the floristic composition of the communities as a whole. Therefore, it was not possible to objectively assess the position of associations in the higher units system of floristic classification based on the analysis performed. To reduce the excessive influence effect of the few main dominants and increase the importance of the floristic criterion, the three species, Quercus pontica, Betula medwediewii and $B$. litwinowii were combined into one combination (into one "pseudo-species") with averaged coverage indices and a cluster analysis (with the same parameters) of the entire series of relevés was performed again. As a result, six clusters (I-VI) interpreted in the previous classification as associations and three higher level clusters (A, B, C) interpreted as alliances were clearly observed on the new dendrogram (Fig. 4) as well. However, at the highest hierarchical level of new dendrogram, all these syntaxa were combined into two completely different clusters (H3 and H4) which according to floristic criteria were defined as two different classes of the Braun-Blanquet system. One association Rhododendro caucasicae-Betuletum litwinowii representing birch krummholz with a predominance of boreal dwarf-shrubs, grasses, mosses and lichens was included in the class Vaccinio-Piceetea

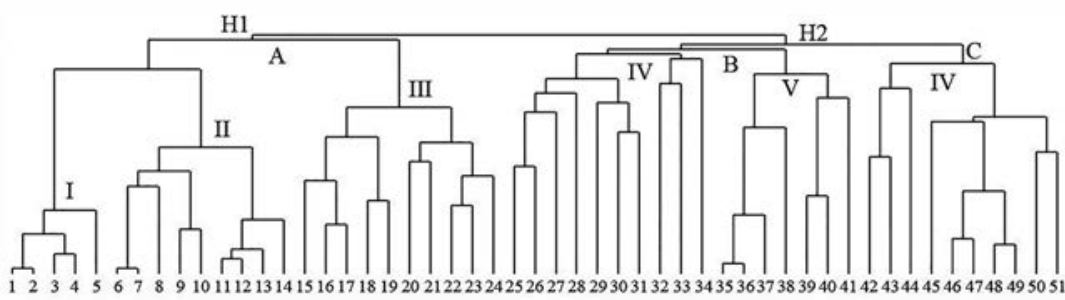

Figure 3 Results of cluster analysis (Ward method, Euclidian distance) of 51 relevés of Caucasian krummholz. Associations: I - Corylo avellanae-Quercetum ponticae ass. nova hoc loco, II - Rhododendro lutei-Quercetum ponticae ass. nova hoc loco, III - Querco ponticaeBetuletum medwediewii Eminagaoglu et al. 2006, IV - Senecioni nemorensis-Betuletum litwinowii Onipchenko 2002, V - Astrantio maximae-Betuletum litwinowii Sokolova ex hoc loco, VI Rhododendro caucasici-Betuletum litwinowii Onipchenko 2002; Alliances: A - Quercion ponticae all. nova. hoc loco, B - Acero trantvetteri-Betulion litwinowii all. nova. hoc loco, $\mathrm{C}-\mathrm{R}$ hododendro caucasici-Betulion litwinowii Onipchenko 2002

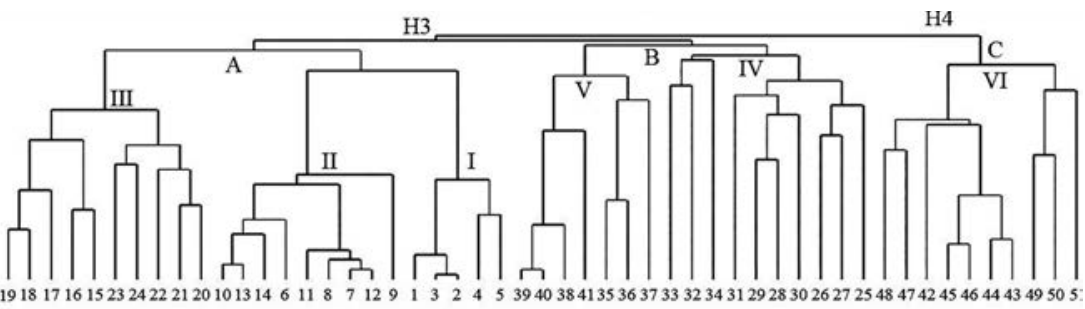

Figure 4 Results of cluster analysis (Ward method, Euclidian distance) of 51 relevés of Caucasian krummholz after reducing the excessive influence effect of the three main dominants - Quercus pontica C. Koch., Betula medwediewii Regel and B. litwinowii Doluch. combined into one combination (into one "pseudo-species") with averaged coverage indices. The designations are the same as in Fig. 3 
Table 1. Associations Corylo avellanae-Quercetum ponticae (relevés 1-5), Rhododendro lutei-Quercetum ponticae (relevés 6-14) and synoptic table of the krummholz syntaxa from the Caucasus (Syntaxa codes: 1s - Corylo avellanae-Ouercetum ponticae, 2s - Rhododendro lutei-Quercetum ponticae, 3s - Querco ponticae-Betuletum medwediewii, 4s - Senecioni nemorensis-Betuletum litwinowii, 5s - Astrantio maximae-Betuletum litwinowii, 6s - Rhododendro caucasici-Betuletum litwinowii). Abbreviations: Cl., Ord., All.Qp transgressive characteristic species of the class Betulo carpaticae-Alnetea viridis, order Acero trantvetteri-Betuletalia litwinowii and alliance Quercion ponticae; s1 - shrub layer (high), s2 - shrub layer (low), hl - herb layer, ml - moss layer.

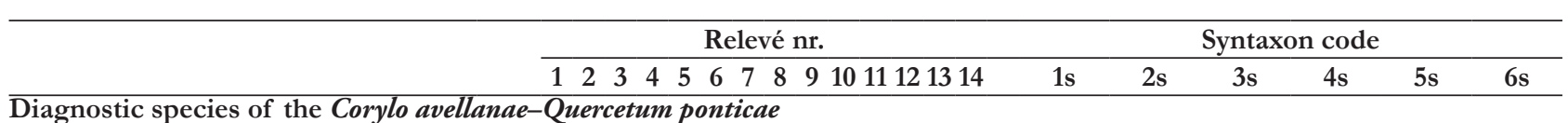

Pulmonaria mollis

Populus tremula

Dentaria bulbifera

Pteridium aquilinum

Polygonatum orientale

hl $\cdot 1+++$.

t1 $2 \begin{array}{llll}2 & 2 & 2 & 2\end{array}$

\begin{tabular}{l|llll} 
hl & + & + & + & + \\
1 & 2 & 1
\end{tabular} .

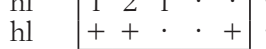

\begin{tabular}{|l|} 
IV \\
IV \\
IV \\
III \\
III \\
\hline
\end{tabular}

Diagnostic species of the Rhododendro lutei-Quercetum ponticae

Adenostyles macrophylla

Aquilegia olympica

Euphorbia oblongifolia

Pulsatilla aurea

Vicia grossheimii

Lilium kesselringianum

Asyneuma campanuloides

\begin{tabular}{llllll|lllllllll|} 
hl & $\cdot$ & $\cdot$ & $\cdot$ & $\cdot$ & $\cdot$ & $\cdot$ & + & + & + & $\cdot$ & 1 & + & 1 & $\cdot$ \\
hl & $\cdot$ & $\cdot$ & $\cdot$ & $\cdot$ & $\cdot$ & + & + & $\cdot$ & + & $\cdot$ & $\cdot$ & $\cdot$ & $\cdot$ & + \\
hl & $\cdot$ & $\cdot$ & $\cdot$ & $\cdot$ & $\cdot$ & $\cdot$ & 1 & 1 & 2 & + & $\cdot$ & $\cdot$ & $\cdot$ & + \\
hl & $\cdot$ & $\cdot$ & $\cdot$ & $\cdot$ & $\cdot$ & $\cdot$ & 1 & + & $\cdot$ & $\cdot$ & + & + & $\cdot$ & $\cdot$ \\
hl & $\cdot$ & $\cdot$ & $\cdot$ & $\cdot$ & $\cdot$ & 1 & + & $\cdot$ & + & + & $\cdot$ & $\cdot$ & $\cdot$ & + \\
hl & $\cdot$ & $\cdot$ & $\cdot$ & $\cdot$ & $\cdot$ & + & $\cdot$ & + & 1 & $\cdot$ & $\cdot$ & + & 1 \\
hl & $\cdot$ & $\cdot$ & $\cdot$ & $\cdot$ & + & + & + & $\cdot$ & $\cdot$ & + & $\cdot$ & $\cdot$ & $\cdot$
\end{tabular}

Diagnostic species of the Querco ponticae-Betuletum medwediewii

Betula medwediewii Cl., Ord., All.Qp.

Rhododendron smirnowii Cl., Ord., All.Qp. st

Epigaea gaultherioides

Scabiosa columbaria

Alopecurus aequalis

Ruscus colchicus

Veronica peduncularis

Sanicula europaea

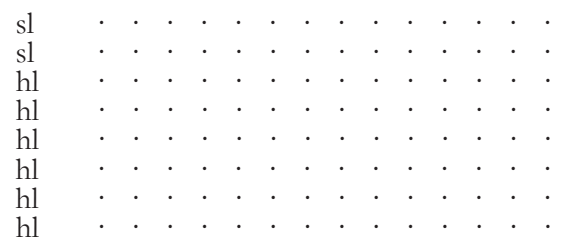

Diagnostic species of the Senecioni nemorensis-Betuletum litwinowii

Heracleum asperum

Cruciata laevipes

Campanula latifolia

Chaerophyllum aureum

Cephalaria gigantea

Brachythecium salebrosum

Stachys macrantha

Dryopteris filix-mas

hl

hl

hl

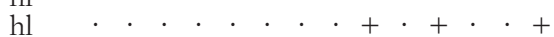

$\mathrm{ml}$

hl

\begin{tabular}{|l|}
\hline IV \\
III \\
III \\
III \\
III \\
III \\
II \\
\hline
\end{tabular}

$\begin{array}{ccc}\text { III } & \cdot & \cdot \\ \text { I } & \cdot & \text { I } \\ \cdot & \cdot & \cdot \\ \cdot & \cdot & \cdot \\ \cdot & \cdot & \cdot\end{array}$

Diagnostic species of the Astrantio maximae-Betuletum litwinowii

Rhododendron caucasicum

Rumex crispus

Festuca drymeja

Inula belenium

Pyrola minor

Euphorbia macroceras

Lilium monadelphum

Hieracium longiscapum

Petasites albus

Poa remota

Epilobium montanum

Myosotis amoena

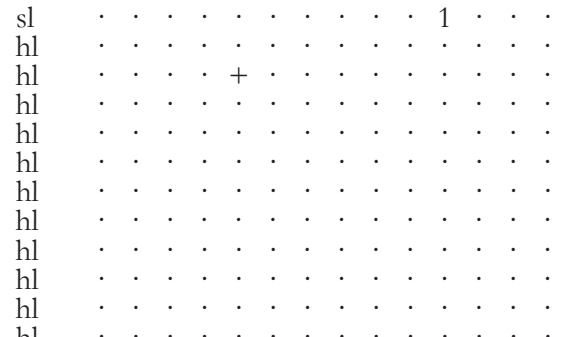

Diagnostic species of the Rhododendro caucasici-Betuletum litwinowii

Dolichorrhiza renifolia

Cladonia pyxidata

Brachythecium reflexum

Vulpicidia pinastri

Hypnum pallescens

$\mathrm{hl}$
$\mathrm{ml}$
$\mathrm{ml}$
$\mathrm{ml}$
$\mathrm{ml}$

Diagnostic species of the Quercion ponticae

Ouercus pontica Cl., Ord.

Rhododendron luteum

Polygonatum verticillatum

Vaccinium arctostaphylos

Corylus avellana

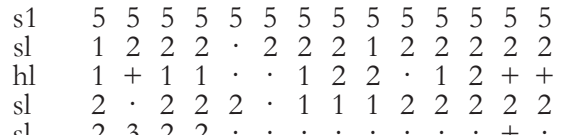

Diagnostic species of the Acero trautvetteri-Betulion litwinowii

Acer trautvetteri $\mathrm{Cl}$., Ord.

Senecio propinquus $\mathrm{Cl}$., Ord

Astrantia maxima Cl., Ord.

Aconitum nasutum

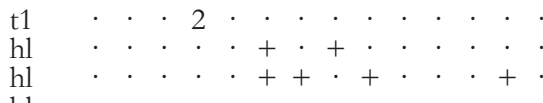

$\mathrm{hl}$

the Betulo carpaticae-Alnetea viridis and Acero trautvetteri-Betuletalia litwinowi

Betula litwinowi

Bistorta carnea

Anemonastrum fasciculatum

Calamagrostis arundinacea

Geranium sylvaticum

Veratrum album

\begin{tabular}{ccccccccccccccc|cccccccc|c|} 
t1 & $\cdot$ & $\cdot$ & $\cdot$ & 2 & 2 & $\cdot$ & $\cdot$ & $\cdot$ & $\cdot$ & $\cdot$ & 2 & 2 & 2 & 2 & II & III & $\cdot$ & V & V & V \\
hl & $\cdot$ & $\cdot$ & $\cdot$ & 2 & $\cdot$ & 2 & 2 & 2 & $\cdot$ & 2 & 2 & 2 & 2 & 2 & I & V & $\cdot$ & I & III & I \\
hl & $\cdot$ & $\cdot$ & $\cdot$ & $\cdot$ & $\cdot$ & 1 & 1 & 2 & $\cdot$ & $\cdot$ & 1 & $\cdot$ & + & 1 & $\cdot$ & IV & $\cdot$ &. & III &. \\
hl & 1 & $\cdot$ & $\cdot$ & 2 & 1 & 2 & 2 & 2 & 2 & + & 2 & 2 & 2 & 2 & III & V & $\cdot$ & V & $\cdot$ & IV \\
hl & $\cdot$ & $\cdot$ & $\cdot$ & + & 1 & 2 & + & + & 1 & $\cdot$ & $\cdot$ & + & + & 1 & II & IV & $\cdot$ & IV & V & II \\
hl & $\cdot$ & $\cdot$ & $\cdot$ & $\cdot$ & $\cdot$ & $\cdot$ & $\cdot$ & $\cdot$ & $\cdot$ & $\cdot$ & $\cdot$ & $\cdot$ & $\cdot$ & $\cdot$ & $\cdot$ & $\cdot$ & $\cdot$ & II & V & I \\
\hline
\end{tabular}


Table 1. Continued.

\begin{tabular}{|c|c|c|c|c|c|c|c|c|c|c|c|c|c|c|c|c|c|}
\hline & & \multicolumn{10}{|c|}{ Relevé nr. } & \multicolumn{6}{|c|}{ Syntaxon code } \\
\hline & & 12 & 23 & 4 & 5 & 67 & 8 & 91 & 1011 & 121 & 1314 & $1 \mathrm{~s}$ & $2 \mathrm{~s}$ & $3 \mathrm{~s}$ & $4 \mathrm{~s}$ & $5 \mathrm{~s}$ & $6 \mathrm{~s}$ \\
\hline \multicolumn{18}{|c|}{ Diagnostic species of the Vaccinio-Piceetea } \\
\hline Vaccinium myrtillus & hl & . & . & . & . & . . & + & & . 1 & 1 & 11 & . & III & II & & III & $\mathrm{V}$ \\
\hline Dicranum scoparium & $\mathrm{ml}$ & . & . . & . & . & . . & . & . & . $\cdot$ & . & . . & . & $\cdot$ & $\cdot$ & I & . & $\mathrm{V}$ \\
\hline Avenella flexuosa & $\mathrm{hl}$ & . & . . & . & . & . . & 2 & . & . 2 & . & . 1 & . & II & . & I & . & IV \\
\hline Juniperus communis & $\mathrm{sl}$ & $\cdot$ & . . & . & . & . . & $\cdot$ & . & $\cdot \cdot$ & $\cdot$ & . . & $\cdot$ & & . & & . & IV \\
\hline Vaccinium vitis-idaea & $\mathrm{hl}$ & . & . . & . & . & . . & $\cdot$ & . & . & . & . . & . & . & $\cdot$ & . & . & IV \\
\hline Hylocomium splendens & $\mathrm{ml}$ & . & . . & . & . & . . & . & . & . & . & . . & . & . & . & I & . & III \\
\hline Cetraria islandica & $\mathrm{ml}$ & . & . . & . & . & . $\cdot$ & $\cdot$ & . & . & . & . & . & . & . & & . & III \\
\hline Gymnocarpium dryopteris & $\mathrm{hl}$ & . & . . & - & . & . $\cdot$ & $\cdot$ & . & . & $\cdot$ & . $\cdot$ & $\cdot$ & - & . & I & . & III \\
\hline Pleurozium scbreberi & $\mathrm{ml}$ & . & . . & . & . & . . & $\cdot$ & . & . & . & . . & . & . & . & I & . & II \\
\hline Empetrum nigrum & $\mathrm{hl}$ & . & . . & . & . & . . & $\cdot$ & . & . & $\cdot$ & . . & . & . & . & . & . & II \\
\hline Barbilophozia barbata & $\mathrm{ml}$ & . & . . & . & . & . . & $\cdot$ & . & . & . & . . & . & . & . & . & . & II \\
\hline Barbilophozia lycopodioides & $\mathrm{ml}$ & . & . $\cdot$ & . & . & . . & $\cdot$ & . & . & . & . & $\cdot$ & . & $\cdot$ & . & . & II \\
\hline Linnaea borealis & $\mathrm{hl}$ & . & . $\cdot$ & . & . & . $\cdot$ & $\cdot$ & . & 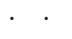 & . & . & 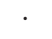 & . & . & . & . & I \\
\hline Peltigera canina & $\mathrm{ml}$ & . & . & . & . & . $\cdot$ & $\cdot$ & . & . & . & . & . & . & . & I & . & I \\
\hline \multicolumn{18}{|l|}{ Other constant species } \\
\hline Sorbus aucuparia & sl & . & $\cdot 2$ & & 2 & $\cdot \cdot$ & & & 2 & & 2 & II & II & $H$ & IV & $\mathrm{V}$ & IV \\
\hline Lapsana communis & $\mathrm{hl}$ & . & . & 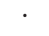 & . & . $\cdot$ & . & & & . & & & & II & III & & \\
\hline Poa nemoralis & $\mathrm{hl}$ & . & . & . & . & . . & . & . & 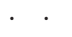 & $\cdot$ & . & . & . & $\mathrm{I}$ & IV & III & I \\
\hline Rubus idaeus & $\mathrm{sl}$ & . & . & . & . & . . & . & . & . & . & . & . & 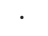 & & IV & $\mathrm{V}$ & II \\
\hline Oberna behen & hl & . & . . & + & . & . . & + & & + & . & . & I & II & I & III & $\mathrm{V}$ & \\
\hline Oxalis acetosella & $\mathrm{hl}$ & . & . & & . & & . & & & . & . . & & & I & $\mathrm{I}$ & $\mathrm{V}$ & $\mathrm{V}$ \\
\hline Solidago virgaurea & $\mathrm{hl}$ & . & . & . & + & . . & . & & . & . & . & I & . & II & I & $\mathrm{V}$ & I \\
\hline Sanionia uncinata & $\mathrm{ml}$ & . & . & . & . & . . & . & & . & . & . & & . & & III & & IV \\
\hline Chamaenerion angustifolium & $\mathrm{hl}$ & . & . & . & . & . . & $\cdot$ & & . & . & & . & . & I & II & . & III \\
\hline
\end{tabular}

Rare species in relevés 1-14: Anthriscus sylvestris $(\mathrm{hl})-8(+), 9(+)$, Aruncus vulgaris $(\mathrm{hl})-14(\mathrm{r})$, Athyrium filix-femina $(\mathrm{hl})-1(+), 14(+)$, Campanula rapunculoides $(\mathrm{hl})-9(+)$, Cervaria aegopodioides $(\mathrm{hl})-9(+)$, Clinopodium vulgare $(\mathrm{hl})-14(+)$, Dianthus cretaceus $(\mathrm{hl})-6(+)$, Fagus orientalis (t1) $-1(2)$, Inula orientalis $(\mathrm{hl})-14(+)$, Kemulariella cancasica $(\mathrm{hl})-11(+), 13(+)$, Linum bypericifolium $(\mathrm{hl})-8(+), 14(+)$, Melampyrum caucasicum $(\mathrm{hl})-11(+), 13(1), 14(+)$, Milium effusum $(\mathrm{hl})-1(+)$, Polygonatum multiflorum $(\mathrm{hl})-2(+)$, Potentilla micrantha $(\mathrm{hl})-4(1), 5(+)$, Primula acaulis $(\mathrm{hl})-8(+)$, Psoralea acaulis $(\mathrm{hl})-9(+)$, Ptarmica biserrata $(\mathrm{hl})-14(+)$, Ranunculus caucasicus $(\mathrm{hl})-4(+), 11(\mathrm{r})$, Rosa canina $(\mathrm{sl})-3$ $(+)$, Rubus caesius $(\mathrm{sl})-2(2), 12(+)$, Sedum stoloniferum $(\mathrm{hl})-9(\mathrm{r})$, Sorbus colcbica $(\mathrm{sl})-7(+), 12(2)$, S. velutina $(\mathrm{sl})-9(+) 11(+)$, Stellaria bolostea $(\mathrm{hl})-9(+)$, Trifolium canescens $(\mathrm{hl})-9(+)$, Vicia balansae $(\mathrm{hl})-9(+), V$. crocea $(\mathrm{hl})-5(+)$. All relevés $(1-14)$ of new associations were made in the upper part of the Pskhu River Basin, Western Caucasus, Abkhasia, 28.07.2019, author - Nikolai Ermakov.

Table 2. Header data of relevés of the associations Corylo avellanae-Quercetum ponticae and Rhododendro lutei-Quercetum ponticae represented in table 1 (rel. 1-14). 1 - relevé number, 2 - relevé area $\left(\mathrm{m}^{2}\right), 3$ - altitude (m a.s.l.), 4 - aspect (degrees), 5 slope (degrees), 6 - cover tree layer (\%), 7 - cover shrub layer (\%), 8 - cover herb layer (\%), 9 - height (highest) trees (m), 10 - height (highest) shrubs (m), 11 - aver. height (high) herbs (cm), 12 - maximum height herbs $(\mathrm{cm}), 13$ - field relevé number, 14 - longitude, 15 - latitude.

\begin{tabular}{|c|c|c|c|c|c|c|c|c|c|c|c|c|c|c|}
\hline 1 & 2 & 3 & 4 & 5 & 6 & 7 & 8 & 9 & 10 & 11 & 12 & 13 & 14 & 15 \\
\hline 1 & 100 & 1780 & 210 & 8 & 10 & 85 & 7 & 19 & 3 & 20 & 35 & 127NE19 & $40^{\circ} 52^{\prime} 26.7^{\prime \prime}$ & $43^{\circ} 25^{\prime} 47.2^{\prime \prime}$ \\
\hline 2 & 100 & 1806 & 170 & 20 & 10 & 90 & 7 & 18 & 2.4 & 20 & 30 & 124NE19 & $40^{\circ} 52 ' 24.2 "$ & $43^{\circ} 25 ’ 50.5^{\prime \prime}$ \\
\hline 3 & 100 & 1814 & 180 & 12 & 15 & 90 & 3 & 19 & 2.7 & 18 & 30 & 125NE19 & $40^{\circ} 52 ' 23.8 "$ & $43^{\circ} 25,50.7 "$ \\
\hline 4 & 100 & 1875 & 210 & 15 & 20 & 90 & 10 & 16 & 2.4 & 25 & 40 & 123NE19 & $40^{\circ} 52 ' 23.0 "$ & $43^{\circ} 25^{\prime} 56.8^{\prime \prime}$ \\
\hline 5 & 100 & 1805 & 97 & 10 & 10 & 95 & 3 & 18 & 2.8 & 20 & 25 & 129NA19 & $40^{\circ} 52^{\prime} 26.1 "$ & $43^{\circ} 25^{\prime} 46.8^{\prime \prime}$ \\
\hline 6 & 100 & 2087 & 150 & 60 & 0 & 95 & 20 & 0 & 1.6 & 20 & 30 & 119NE19 & $40^{\circ} 51 ’ 16.3 \prime$ & $43^{\circ} 27^{\prime} 12.1^{\prime \prime}$ \\
\hline 7 & 100 & 2120 & 180 & 65 & 0 & 98 & 20 & 0 & 1.8 & 20 & 35 & 128NE19 & $40^{\circ} 51 ' 12.1 "$ & $43^{\circ} 27^{\prime} 09.1^{\prime \prime}$ \\
\hline 8 & 100 & 2131 & 120 & 60 & 0 & 95 & 25 & 0 & 1.6 & 25 & 40 & 115NE19 & $40^{\circ} 52^{\prime} 44.5 \prime$ & $43^{\circ} 26 ' 13.8^{\prime \prime}$ \\
\hline 9 & 100 & 2125 & 150 & 35 & 0 & 98 & 15 & 0 & 1.5 & 25 & 35 & 117NE19 & $40^{\circ} 52 ' 48.6 "$ & $43^{\circ} 26 ' 14.3$ " \\
\hline 10 & 100 & 2098 & 200 & 30 & 0 & 95 & 7 & 0 & 1.5 & 20 & 35 & 118NE19 & $40^{\circ} 52 ’ 38.8 "$ & $43^{\circ} 26 ' 12.1 "$ \\
\hline 11 & 100 & 2085 & 220 & 30 & 0 & 95 & 20 & 0 & 1.6 & 20 & 25 & 121NE19 & $40^{\circ} 52^{\prime} 37.9 "$ & $43^{\circ} 26 ' 10.8^{\prime \prime}$ \\
\hline 12 & 100 & 2120 & 130 & 60 & 0 & 95 & 15 & 0 & 2 & 30 & 40 & 116NE19 & $40^{\circ} 52^{\prime} 43.0^{\prime \prime}$ & $43^{\circ} 26 ' 12.8^{\prime \prime}$ \\
\hline 13 & 100 & 2080 & 190 & 25 & 0 & 95 & 15 & 0 & 2.5 & 15 & 25 & 122NE19 & $40^{\circ} 53 ' 29.1 "$ & $43^{\circ} 26^{\prime} 05.1 "$ \\
\hline 14 & 100 & 2090 & 90 & 50 & 0 & 95 & 18 & 0 & 2 & 20 & 30 & 120NE19 & $40^{\circ} 53 ' 46.7 \prime$ & $43^{\circ} 25 ' 55.3^{\prime \prime}$ \\
\hline
\end{tabular}

(H4). All remaining five associations (I-V) of subalpine oak and birch krummholz with tall-forbs and nemoral species were included in the class Betulo-Alnetea viridis (H3).

\section{Characterization of associations}

Corylo avellanae-Quercetum ponticae ass. nova hoc loco (Tables 1, 2, relevés $1-5$ )

Holotypus: relevé 2, Table 1.

Diagnostic species: Populus tremula, Pulmonaria mollis, Dentaria bulbifera, Pteridium aquilinum, Polygonatum orientale.

Description. This association occurs in the upper part of the forest belt near the border with the subalpine one at altitudes 1700-1900 m a.s.l. Small dense patches of this community occur at the tops of the mountains where they occupy gentle $\left(5-15^{\circ}\right)$ parts of the southern, southeastern and southwestern slopes with poorly developed soils.

The open tree layer (with a cover of $10 \%$ and a height 4-16 m) is formed by Populus tremula. Quercus pontica with a mix of Corylus avellana and Rhododendron luteum form close (85-90 \%) 2-3 m high shrub layer.

The grass layer is sparse (5-15\% coverage) because of strong shading. The poor species composition of the community consists mainly of hemiboreal and nemoral species (Pulmonaria mollis, Pteridium aquilinum, Vaccinium arctostaphylos, 
Dentaria bulbifera, Polygonatum verticillatum, P. orientale). Subalpine tall-forb plants are single. The moss layer is absent.

Rhododendro lutei-Quercetum ponticae ass. nova hoc loco (Tables 1, 2, relevés 6-14).

Holotypus: relevé 7 , Table 1.

Diagnostic species: Adenostyles macrophylla, Aquilegia olympica, Euphorbia oblongifolia, Pulsatilla aurea, Vicia grossheimii, Lilium kesselringianum, Asyneuma campanuloides.

Description. The association includes subalpine krummholz with absolute predominance of Quercuspontica occurring at the upper boundary of the forest belt at altitudes of $1800-2150 \mathrm{~m}$. They were found as small patches on very steep $\left(45-60^{\circ}\right)$ slopes of the southern, south-western and south-eastern aspects with poorly developed rocky soils.

Quercus pontica forms a very dense 2-3 m high shrub layer with a cover $90-100 \%$. The small trees of Betula litwinowi and shrubs (Rhododendron luteum) occur there as well. High cover values of the upper layer causes unfavorable shading for the lower plants. Therefore, the grass layer has values of cover 5-15\% and species richness 5-8 species per $100 \mathrm{~m}^{2}$ ). Endemic Caucasian tall-forb species (Adenostyles macrophylla, Lilium kesselringianum, Asyneuma campanuloides, Cephalaria gigantea, Astrantia maxima, Bistorta carnea, Anemonastrum fasciculatum) and widespread European species (Calamagrostis arundinacea, Geranium sylvaticum) prevail there. The moss layer is not developed.

Note. A similar Quercus pontica community (association Querco ponticae-Betuletum medwediewii Eminagaoglu et al. 2006) (Table 1, Syntaxon 3s) was described from the upper part of the Lesser Caucasus forest belt (Eminagaoglu et al. 2006). According to our concept, diagnostic species of this association are Betula medwediewii, Rhododendron smirnowii, Epigaea gaultherioides, Scabiosa columbaria, Alopecurus aequalis, Ruscus colchicus, Veronica peduncularis, Sanicula europaea. This community descends into the forest belt in the form of strips along extrazonal habitats - local areas on steep mountain slopes disturbed periodically by avalanches. As in the Corylo-Quercetum ponticae association, the upper open tree and dense shrub layers are formed by subalpine species (Quercus pontica and Betula medwediewii) however moderately thermophilous species from surrounding forests prevail in the grass layer.

\section{Astrantio maximae-Betuletum litwinowii Sokolova ex hoc loco (Table 1, Syntaxon 5s)}

Holotypus: relevé 4, Table 1 in Sokolova (2013) (Proceedings of Voronezh State University. Series: Chemistry. Biology. Pharmacy 1, 2013, pp. 170 - 171).

Synonym: Rhododendro caucasicae-Betuletum litwinowii Sokolova prov.

Diagnostic species: Rhododendron caucasicum, Rumex crispus, Festuca drymeja, Inula belenium, Pyrola minor, Euphorbia macroceras, Lilium monadelphum, Petasites albus, Hieracium longiscapum, Poa remota, Epilobium montanum, Myosotis amoena.

Description. The association is spread in the upper reaches of the river Belaya on the northern macro-slope of the Greater Caucasian Ridge (Tybga Mountain, North-Western Caucasus). It occupies the western and south-western slopes at the upper boundary of the forest, at altitudes of 1800-2000 $\mathrm{m}$ a.s.l. The higher layer is formed by Betula litwinowii with a mix of Acer trantvetteri and Sorbus aucuparia (cover 30-60 \% and average height $17 \mathrm{~m}$ ). The shrub layer is dominated by Rhododendron caucasicum (cover 5-40\%). Tall-forb subalpine species (Astrantia maxima, Veratrum lobelianum, Inula belenium, Oberna behen, Poa remota, Anemonastrum fascicula, Bistorta carnea, Senecio propinquus, Petasites albus, Aconitum nasutum, Cicerbita macrophylla, Aconitum orientale) pre- dominate in the well-developed grass layer (cover 70-80\% and height $80-110 \mathrm{~cm}$ ).

\section{Senecioni nemorensis-Betuletum litwinowii \\ Onipchenko 2002 (Table 1, Syntaxon 4s)}

Diagnostic species: Heracleum asperum, Cruciata laevipes, Campanula latifolia, Chaerophyllum aureum, Cephalaria gigantea, Brachythecium salebrosum, Stachys macrantha, Dryopteris filix-mas.

Note. The association of Betula litwinowii subalpine krummholz described by Onipchenko (2002) on the territory of the Teberdinsky Biosphere Nature Reserve located on the northern macroslope of the Greater Caucasian Ridge. It is characterized by the absolute predominance of tall-forb species and is the widespread typical example of the subalpine krummholz in the Caucasus.

\section{Rhododendro caucasici-Betuletum litwinowii}

Onipchenko 2002 (Table 1, Syntaxon 6s).

Note. The association differs essentially from all communities described above in the almost complete absence of subalpine tall-forb and nemoral herb species. In this community occupying colder and wetter habitats on the shaded slopes of the mountains an absolute predominance of boreal dwarf shrubs (Vaccinium myrtillus, V. vitis-idaea, Empetrum nigrum), herbs (Avenella flexuosa, Gymnocarpium dryopteris, Linnaea borealis), lichens and mosses (Hylocomium splendens, Pleurozium schreberi, Dicranum scoparium, Barbilophozia barbata, B. Lycopodioides, Peltigera canina, Cetraria islandica) is observed. The moss layer of the community is usually well developed.

\section{DISCUSSION}

The uniqueness of the high mountain flora and vegetation of the Caucasus is determined by the high level of endemism (Kolakovsky 1961, Gagnidze 1974, Shetekauri et al. 2012, Ermakov et al. 2019). The authors who developed local classifications of communities formed by endemic subalpine species of low trees and high shrubs (Betula litwinowii, B. medwediewii, Quercus pontica) (Onipchenko 2002, Eminagaoglu et al. 2006, Sokolova 2013) represented completely different opinions on the positions of described syntaxa in the Braun-Blanquet higher units system. Eminagaoglu et al. (2006) assigned the association Querco ponticae-Betuletum medwediewii to broad-leaved forests of the class Carpino-Fagetea Jakucs ex Passarge 1968 (order Pino-Piceetalia orientalis Quezel et al. 1980, alliance Geranio-Pinion sylvestris Quezel et al. 1980). However, this decision is unconvincing because among the characteristic species of the broadleaved forests class only Corylus avellana and Sanicula europaea have high constancy values (Table 1). At the same time, typical species of the subalpine flora (Quercus pontica, Betula medwediewii, Rhododendron smirnowii) demonstrate not only highest constancy values but also are dominants. According to the cluster analysis results, the associations Querco ponticae-Betuletum medwediewii, Rhododendro lutei-Quercetum ponticae and Corylo-Quercetum ponticae were combined into one cluster (A) of a higher hierarchical level (Fig. 3, 4) interpreted as the alliance Quercion ponticae all. nova hoc loco. Holotypus of this alliance is the association Rhododendro lutei-Quercetum ponticae ass. nova hoc loco. Diagnostic species of the alliance are Quercus pontica, Betula medwediewi, Rhododendron smirnowii, $\mathrm{R} h$. luteum, Vaccinium arctostaphylos, Corylus avellana and Polygonatum verticillatum. Majority of these species are endemics of Caucasian arboreal flora. The Quercion ponticae communities occur in the most humid high 
mountains of the South-Western Caucasus where they are found exclusively on well-insulated very steep parts of the southern mountain slopes. The predominance of Quercus pontica provides the main common phytocenotic feature of the alliance communities - a dense higher layer which ultimately results in the intensive shading and poor floristic composition of the ground layer.

In contrast, the Betula litwinowii krummholz of the associations Senecioni nemorensis-Betuletum litwinowii and Astrantio maximae-Betuletum litwinowii are characterized by a welldeveloped herb layer consisting of the numerous lightdemanding tall-forb species. This floristic feature determined the integration of these associations into one separate cluster (B) in Fig. 3, 4. The relevés of the association Rhododendro-Betuletum litwinowii Onipchenko 2002 also separated as a special cluster $(C)$ at the highest hierarchical level in the dendrograms (Fig. 3, 4).

The results of cluster analysis contradict currently existing interpretations of the position of the last three associations in the Braun-Blanquet higher units system, which were presented in Onipchenko (2002), Sokolova (2013), Mucina et al. (2016). The associations Rhododendro-Betuletum litwinowii and Senecioni nemorensis-Betuletum litwinowii united by Onipchenko (2002) in the alliance Rhododendro-Betulion litwinowii Onipchenko 2002 were dispersed into different clusters (Fig. 3, 4) because they differ significantly in floristic compositions. The first association, Rhododendro-Betuletum litwinowii chosen as "typus" (holotypus) for the alliance is characterized by predominance of boreal plants in the ground layer (Vaccinium myrtillus, V. vitis-idaea, Linnaea borealis, Empetrum nigrum, Avenella flexuosa, Gymnocarpium dryopteris, Pleurozium schreberi, Dicranum scoparium, Hylocomium splendens, Barbilophozia barbata, B. lycopodioides, Cetraria islandica, Peltigera canina) and occupies colder sites. Therefore, the alliance Rhododendro-Betulion litwinowii was placed originally by Onipchenko (2002) in the order Piceetalia excelsae Pawłowski et al. 1928 and class Vaccinio-Piceetea Br.-Bl. в Br.-Bl. al. 1939.

The second association Senecioni nemorensis-Betuletum litwinowii Onipchenko 2002 occurring in warmer sites and uniting birch krummholz with predominance of subalpine tall-forb species was also included in the alliance Rhododendro-Betulion litwinowii. However, Onipchenko (2002) noted that this syntaxonomic solution requires further refinement, since it lacks boreal species. In accordance with the results of our analysis, it becomes obvious that these two associations differ significantly in floristic compositions, characterize two large ecological types of communities, and must be attributed to different higher syntaxonomic units. However, Mucina (Mucina et al. 2016), based on data published by Onipchenko (2002), mistakenly included the alliance Rhododendro-Betulion litwinowii and new order Rhododendro caucasici-Betuletalia litwinowii Mucina 2016 in the class Betulo carpaticae-Alnetea viridis Rejmanek ex Boeuf et al. 2014 despite the fact that the characteristic species of the boreal class Vaccinio-Piceetea prevail in the holotypus of this alliance (association Rhododendro caucasicae-Betuletum litwinowii Onipchenko 2002). Based on the results of the quantitative classification and comparative syntaxonomic analysis, we propose another concept for the Caucasian subalpine krummholz classification. The association Rhododendro caucasicae-Betuletum litwinowii, alliance Rhododendro caucasicae-Betulion litwinowii Onipchenko 2002 and order Rhododendro caucasici-Betuletalia litwinowii Mucina 2016 should be included in the Vaccinio-Piceetea in accordance with the mentioned above fact of the absolute predominance of diagnostic species of this class (Table 1). As a result, it becomes obvious that the previously proposed (Mucina et al. 2016) order of the subalpine-subarctic birch krummholz of Europe, the Vaccinio myrtilli-Betuletalia pubescentis Mucina et Willner 2016 (prov.) and the Caucasian order Rhododendro caucasici-Betuletalia litwinowii Mucina 2016 represent vicarious types of subalpine and subarctic krummholz with boreal floristic elements and they may be considered as synonyms. The validly published order Rhododendro caucasici-Betuletalia litwinowii has a priority name and can represent geographically wide category including all Eurasian subalpine and subarctic krummholz with predominance of boreal elements (within the class Vaccinio-Piceetea).

The associations Senecioni nemorensis-Betuletum litwinowii and Astrantio maximae-Betuletum litwinowii representing Caucasian birch krummholz with participation of subalpine tall-forbs should be included in the new alliance Acero trantvetteri-Betulion litwinowii nova hoc loco. Holotypus of this alliance is the association Senecioni nemorensis-Betuletum litwinowii Onipchenko 2002. Diagnostic species of the alliance are Acer trautvetteri, Senecio propinquus, Astrantia maxima, Aconitum nasutum. These communities are widespread in subalpine belt of the Caucasus at altitudes 1900-2300 m. The alliances Acero trautvetteri-Betulion litwinowii and Quercion ponticae were included in the new order Acero trantvetteri-Betuletalia litwinowii ord. nova hoc loco, in the class Betulo-Alnetea viridis. Holotypus of this order is the alliance Acero trautvetteri-Betulion litwinowii all. nova hoc loco. The Acero trautvetteri-Betuletalia litwinowii represents all communities of Caucasian subalpine krummholz with tall-forbs and it differs well floristically from similar orders described in Central European mountain systems due to the group of endemic Caucasian plants (Betula litwinowii, B. medwediewii, Quercus pontica, Acer trantvetteri, Rhododendron smirnowii, Astrantia maxima, Anemonastrum fasciculatum, Aconitum orientale, Bistorta carnea, Senecio propinquns). These typical subalpine species are characteristic taxa for the Acero trantvetteriBetuletalia litwinowii and regional diagnostic species for the class Betulo carpaticae-Alnetea viridis. The concept of our new proposals concerning the classification of Caucasian krummholz is given in the classification below.

Classification of the Caucasian subalpine krummholz vegetation

Class

Order

Alliance

Association

Betulo carpaticae-Alnetea viridis Rejmanek ex Boeuf, Theurillat, Willner, Mucina et Simler in Boeuf et al. 2014

Acero trautvetteri-Betuletalia litwinowii ord. nova hoc loco

Acero trautvetteri-Betulion litwinowii all. nova hoc loco

Senecioni nemorensis-Betuletum litwinowii

Onipchenko 2002 


\section{Astrantio maximae-Betuletum litwinowii}

Sokolova ex hoc loco (Rhododendro caucasici-Betuletum

litwinowii Sokolova 2016 prov., syntax. synonym)

Quercion ponticae all. nova hoc loco

Rhododendro lutei-Quercetum ponticae ass. nova hoc loco

Corylo avellanae-Quercetum ponticae ass. nova hoc loco

Querco ponticae-Betuletum medwediewii

Eminagaoglu et al. 2006

Vaccinio-Piceetea Br.-Bl. in Br.-Bl. et al. 1939

Rhododendro caucasici-Betuletalia litwinowii Mucina 2016 (Vaccinio myrtilli-Betuletalia pubescentis Mucina et Willner 2016 prov., syntax. synonym).

\section{Rhododendro caucasici-Betulion litwinowii}

Onipchenko 2002

Rhododendro caucasici-Betuletum litwinowii Onipchenko 2002

\section{CONCLUSION}

A new classification system of the subalpine krummholz vegetation of the Caucasus based on the geographically extensive data set has been developed. Two communities of a unique endemic species Quercus pontica were described as associations Rhododendro lutei-Quercetum ponticae ass. nova hoc loco and Corylo avellanae-Quercetum ponticae ass. nova hoc loco, which together with the association Querco ponticae-Betuletum medwediewii Eminagaoglu et al. 2006 were united in a new alliance Quercion ponticae all. nova hoc loco. All these communities are characterized by a high degree of shading from the closed higher layer resulting in a poor floristic composition of the ground layer. In contrast, the widespread less closed Betula litwinowii subalpine krummholz are characterized by well-developed herb layer dominated by the light-demanding subalpine tall-forb species. Two associations of these birch krummholz (Senecioni-Betuletum litwinowii Onipchenko 2002 and Astrantio-Betuletum litwinowii Sokolova ex hoc loco) were united in the alliance Acero trantvetteri-Betulion litwinowii all. nova hoc loco. The latter together with another alliance Quercion ponticae were included in the order Acero trautvetteri-Betuletalia litwinowii ord. nova hoc loco (the class Betulo-Alnetea viridis Rejmanek ex Boeuf et al. 2014). The peculiarity of this order is determined by a high level of Caucasian endemism in the floristic composition which distinguishes it well from other orders representing similar communities in mountains of Europe. Another ecological and floristic type of subalpine krummholz in the Caucasus is Betula litwinowii community with predominance of boreal shrubs and mosses in the ground layer occupying colder habitats on the shaded mountain slopes (alliance Rhododendro-Betuletion litwinowii Onipchenko 2002, order Rhododendro caucasicae-Betuletalia litwinowii Mucina 2016). It was included in the VaccinioPiceetea Br.-Bl. in Br.-Bl. et al. 1939. Together with boreal pine (Pinus sylvestris var. hamata) forests of the same class (Ermakov et al. 2019) they represent examples of relic Pleistocene communities occurring locally in the subalpine belt in northern macro-slope of the Greater Caucasus. All these new syntaxonomic proposals are important for the further development of the subalpine higher units system adopted for European Vegetation Classification (Mucina et al. 2016).

\section{ACKNOWLEDGEMENTS}

The study was supported by Russian Foundation for Basic Researches (grants 18-04-00633, 19-54-40005 Абx_a).

\section{LITERATURE CITED}

Antonov, B.A., N.V. Dumitrashko, N.Sh. Shirinov 1977. General characteristics and history of the relief development of the Cancasus. Nauka, Moscow, 288 pp. (in Russian). [Антонов Б.А., Аумитрашко Н.В., Ширинов Н.Ш. 1977. Общая характеристика и история развития рельефа Кавказа. Москва: Наука. 288 с.].

Czerepanov, S.K. 1995. Vascular plants of Russia and adjacent states (the former USSR). Cambridge University Press, Cambridge, 516 pp.

Eminagaoglu, O., H. Kutbay, A. Bilgin, E. Yalcin 2006. Contribution to the phytosociology and conservation of Tertiary relic species in North-Eastern Anatolia (Turkey). Belgian Journal of Botany 139(1):124-130.

Ermakov, N.B., Z.I. Abdurakhmanova, I.L. Potapenko 2019. To the problem of syntaxonomy of pine forests (Pinus sylvestris var. hamata) with the participation of boreal floristic elements in Dagestan (North Caucasus). Turczaninowia 22(4):154-171 (in Russian with English abstract). [Ермаков Н.Б., Абаурахманова 3.И., Потапенко И.А. 2019. К проб̆еме синтаксономии сосновых месов (Pinus sylvestris var. hamata) с участием бореальных флористических элементов в Аагестане (Северный Кавказ) // Turczaninowia. Т. 22, № 4. С. 154-171].

Gagnidze, R.I. 1974. Plant-geographical analysis of floro-coenotic complex of subalpine tall-forb vegetation of the Caucasus. Metsniereba, Tbilisi, 226 pp. (in Russian). ГГагнилзе Р.И. 1974. Ботанико-географический анализ флороценотического комплекса субальпийского высокотравья Кавказа. Тбилиси: Мецниереба. 226 с.].

Grossheim, A.A. 1936. Analysis of Caucasian Flora. In: Proceedings of the Botanical Institute of the Azerbaijan Branch of Academy of Science of the USSR, vol. 1. Izdatel'stvo Azerbaidzhanskogo filiala AN SSSR, Baku, 259 pp. (in Russian). [Гроссгейм А.А. 1936. Анализ флоры Кавказа / / Труды Ботанического ин-та Азербайджанского филиала АН СССР. Т. 1, Баку: ИзА-во АзербайАжанского ф-ла AH CCCP. 259 c.].

Grossheim, A.A. 1948. Vegetation cover of the Caucasus. Izdatel'stvo Moskovskogo Obshchestva Ispytatelei Prirody, Moscow, 268 pp. (in Russian). [Гроссгейм А.А. 1948. Растительный покров Кавказа. Москва: ИзА-во МОИП. 268 с.].

Gulisashvili, V.Z., L.B. Makhatadze, L.I. Prilipko 1975. Vegetation of the Caucasus. Nauka, Moscow, 233 pp. (in Russian). [Гулисашвили В.3., Махатадзе А.Б., Примипко А.И. 1975. Растительность Кавказа. Москва: Наука. 233 с.].

Hennekens, S.M. \& J.H.J. Schaminée 2001. Turboveg, a comprehensive data base management system for vegetation data. Journal of Vegetation Science 12:589-591.

Ignatov, M.S., O.M. Afonina, E.A. Ignatova, A. Abolina, T.V. Akatova, E.Z. Baisheva, L.V. Bardunov et al. 2006. Check-list of mosses of East Europe and North Asia. Arctoa 15:1-130.

Kamanin, L.G., P.N. Tsys, N.S. Blagovolin 1974. Mountain systems of the European part and the Caucasus. Nauka, Moscow, 360 pp. (in Russian). [Каманин А.Г., Цысь П.Н., БАаговолин Н.С. 1974. Горные страны Европейской части и Кавказ. Москва: Наука. 360 с.]. 
Kolakovsky, A.A. 1961. Plant life of the Kolkhida. In: Materials for the knowledge of the fauna and flora of the USSR, published by the Moscow Society of Naturalists. New Series, Botanical Department 10 (XVIII). Izdatel'stvo Moskovskogo gosudarstvennogo universiteta, Moscow, 460 pp. (in Russian). [Колаковский А.А. 1961. Растительный мир Колхиды / / Материалы к познанию фауны и флоры СССР, издаваемые Московским обществом испытатемей природы. Новая серия, ОтАел ботанический 10 (XVIII). Москва: Иза-во МГУ. 460 с.].

Kolakovsky, A.A. 1982. Flora of Abkhasia. Vol. II. Magnoliophyta-Labiatae. Mencniereba, Tbilisi, 282 pр. [Колаковский A.А. 1982. Фмора Абхазии. Magnoliophyta-Labiatae. Тбимиси: ИзА-во Мецниереба. Т. 2. 282 с.].

McCune, B. \& M.J. Mefford 2006. PC-ORD. Multivariate Analysis of Ecological Data. Version 6. MjM Software, Gleneden Beach, Oregon, USA. 237 pp.

Mucina, L., H. Bultmann, K. Dierßen, J.-P. Theurillat, T. Raus, A. Carni, K. Sumberova et al. 2016. Vegetation of Europe: hierarchical floristic classification system of vascular plant, bryophyte, lichen, and algal communities. Applied Vegetation Science 19(1):3-264.

Onipchenko V.G. 2002. Alpine vegetation of the Teberda Reserve, the Northwestern Caucasus. Geobotanisches Institut ETH, Stiftung Rubel, Zurich, 168 pp.

Shetekauri, S., D. Chelidze \& N. Barnaveli 2012. Diversity and floro-genesis of subnival flora of the Caucasus. Journal of Life Sciences 6:917-931.

Sokolova, T.A. 2013. Syntaxonomy of high mountain forests of the North-Eastern Caucasus. Vestnik Voronezhskogo gosudarstvennogo Universiteta. Seriya: Khimiya. Biologiya. Pharmaciya 1: 170-171 (in Russian). [Соколова T.A. 2013. Синтаксономия растительности высокогорных цесов Северо-Западного Кавказа // Вестник ВГУ, Серия: Химия. Биология. Фармация. №1. С. 166-176.].

Tephnadze, N., O. Abdaladze, G. Nakhutsrishvili, D. Simmering, R. Waldhardt \& A. Otte 2014. The impacts of management and site conditions on the phytodiversity of the upper montane and subalpine belts in the Central Greater Caucasus. Phytocoenologia 44(3-4):255-291.

Tumadzhanov, I.I. 1980. High mountain meadows and open forests. In: Vegetation of European part of the USSR (S.A. Gribova et al., eds.), pp. 198-202, Nauka, Leningrad (in Russian). [Тумаджанов И.И. 1980. Высокогорные муга и криволесья // Растительность Европейской части СССР / под реА. С.А. Грибовой и Ар. АенинграА: Наука. С. 198-202].

Weber, H.E., J., Moravec \& J.-P. Theurillat 2000. International code of phytosociological nomenclature. 3rd ed. Journal of Vegetation Science 11(5):739-768.

Westhoff, V. \& E. van der Maarel 1973. The BraunBlanquet Approach. In: Ordination and classification of communities (R.H. Whittaker, ed.), pp. 617-626, Dr. W. Junk, Dordrecht.

Zazanashvili, N., R. Gagnidze, G. Nakhutsrishvili 2000. Main types of vegetation zonation on the mountains of the Caucasus. Acta Phytogeographica Suecica 85:7-16. 